\title{
Fechamento de diastemas anteriores com resina composta direta: relato de caso
}

\section{Anterior diastema closure with direct composite resin: case report}

\author{
Micaela Lorena Raposo Seixas Guerra' \\ Gisely NaURA VenÂNCIO" \\ Carolina Rocha Augusto"II
}

\begin{abstract}
RESUMO
Introdução: Os diastemas são espaços interdentais que ocorrem nos arcos superior e inferior, tanto em dentes anteriores como em dentes posteriores, sendo mais frequentes em dentes anterossuperiores. Sua etiologia é multifatorial e deve ser avaliada com cautela para que o tratamento correto seja realizado e concluído com sucesso.

Objetivo: O presente artigo possui como objetivo relatar um caso clínico em que se optou pela confecção de restaurações em resina composta direta para o fechamento dos diastemas.

Caso clínico: Paciente de 21 anos de idade, procurou atendimento no curso de graduação em Odontologia da Universidade Nilton Lins queixando-se do espaço que havia entre seus incisivos superiores. Após exame clínico, foi sugerida a confecção de facetas em resina composta pela técnica direta.

Conclusão: Por meio desse caso clínico conclui-se que a realização de facetas utilizando materiais adesivos destaca-se por reduzido tempo de trabalho e baixo custo. Mostrando ser uma técnica eficaz no restabelecimento dos aspectos funcionais e estéticos do sorriso.
\end{abstract}

Palavras-chave: Diastema. Restauração dentária permanente. Resinas compostas. Estética. Sorriso.

\begin{abstract}
Introduction: A diastema is an interdental space in the upper or lower jaw, in both anterior and posterior teeth, being more frequent in anterosuperior teeth. Its aetiology is multifactorial and should be analysed carefully so that the appropriate treatment is carried out and completed successfully.

Objective: This paper aims to report a case in which it was opted to apply direct composite resin restorations to undertake the closure of a diastema.

Clinical case: Patient of 21 years old, sought care at the undergraduate course in Dentistry, University Nilton Lins, complaining about the existing space between his front teeth. After clinical examination, he was advised to apply composite resin veneer, following the direct technique.

Conclusion: Through this case report, it can be concluded that performing veneers using adhesives stand out for reduced working time and low costs, proving to be an effective technique in restoring the functional and aesthetic aspects of smile.
\end{abstract}

Key-words: Diastema. Permanent dental restoration. Composite resin. Aesthetics. Smile.

Universidade Nilton Lins, Manaus/AM - Brasil.

"Faculdade São Leopoldo Mandic, Campinas/SP - Brasil.

"'Universidade Nilton Lins, Manaus/AM - Brasil. 


\section{INTRODUÇÃO}

Diastema é o espaço, a lacuna ou a ausência de contato entre dois ou mais dentes contíguos. Apesar de poderem ser vistos em qualquer região dos arcos, esses espaços são mais frequentes na região anterossuperior, pelo fato de ocorrer uma discrepância entre o tamanho dos elementos dentários e a maxila. Sua etiologia é multifatorial, portanto, é necessário e fundamental fazer uma avaliação correta de sua origem para que assim seja proposto um tratamento adequado e com sucesso restabelecer as características estéticas faciais. ${ }^{1,2}$

A estética facial e o sorriso estão intimamente relacionados e a procura por um sorriso perfeito e harmonioso teve um aumento bastante significativo. Com isso, pacientes têm manifestado a vontade de obter restaurações com materiais na cor e forma naturais pelo fato de que dentes claros, bem contornados e alinhados, estabelecem o padrão de beleza do indivíduo. Tais dentes não são apenas considerados atraentes, indicam também saúde nutricional, amor-próprio e status, visto que a necessidade estética em um tratamento odontológico é hoje uma exigência e deve ser considerada tão importante quanto a recuperação funcional de um elemento dentário. $^{3}$

Para a correção dos diastemas, são indicados os tratamentos ortodônticos, facetas diretas em resina composta, facetas de porcelanas, lentes de contato, coroas cerâmicas, entre outros. ${ }^{1,2}$ Nos casos de espaçamento maior que $3,0 \mathrm{~mm}$ de largura, o tratamento ortodôntico é o mais indicado, enquanto para diastemas variando de $0,5 \mathrm{~mm}$ a 3,0 $\mathrm{mm}$ de largura o fechamento com resina composta pode ser realizado. ${ }^{4}$

Uma das vantagens que mais se destacam nas resinas compostas trata do resultado estético, pois com esse material é possível recriar uma aparência natural próxima às estruturas dentais, restaurando a função morfológica e restabelecendo características como cor, translucidez, matiz, croma e valor. ${ }^{5}$

A técnica de restaurações diretas para remodelação em resina composta consiste na aplicação e escultura de uma ou mais camadas desse material sobre a superfície do elemento dentário, sendo considerado um procedimento menos invasivo, possibilitando assim uma conservação maior de estrutura dental sadia, resultando em um sorriso harmonioso e estético. ${ }^{6}$

Dessa maneira, o presente artigo possui como objetivo relatar um caso clínico em que se optou pela confecção de facetas em resina composta direta para o fechamento de diastemas na região anterossuperior, nos elementos 11, 21 e 22. Deste modo, dando ênfase ao planejamento e execução da técnica com a finalidade em melhorar a estética e harmonia do sorriso, devolvendo bem-estar e autoestima à paciente de maneira conservadora e atual.

\section{Caso clínico}

Paciente leucoderma, gênero feminino, 21 anos de idade, procurou atendimento no curso de graduação em Odontologia da Universidade Nilton Lins, na cidade de Manaus$\mathrm{AM}$, queixando-se do espaço que havia entre seus incisivos superiores. Após anamnese e exames clínico e físico detalhados, foi constatado que a paciente tinha diastemas anterossuperiores de $1 \mathrm{~mm}$ nos elementos 11, 21 e 22. Foi sugerido um tratamento interdisciplinar ortodôntico-restaurador, no entanto, a mesma recusou, pois queria uma alternativa mais rápida e menos onerosa. Como segunda opção, foi proposta a confecção de restaurações em resina composta.

O registro fotográfico inicial foi realizado (Figura 1). 


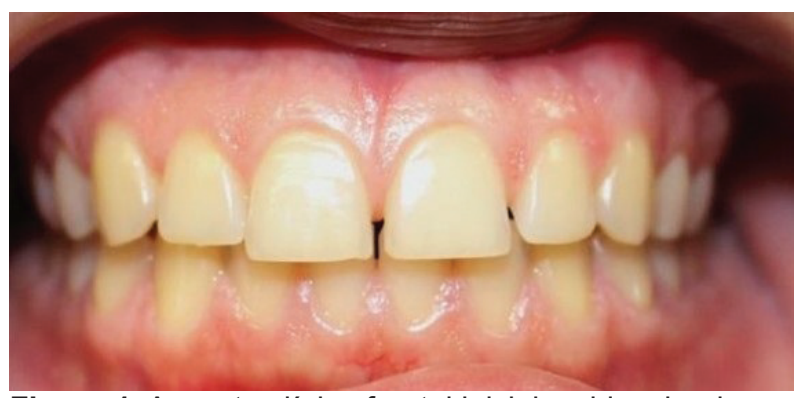

Figura 1. Aspecto clínico frontal inicial, evidenciando os diastemas

Para a obtenção dos modelos de estudo, os arcos superior e inferior foram moldados com alginato tipo I (Jeltrate Plus, Dentsplay, Brasil) e vazados com gesso especial (Vênus tipo IV, Yamay, Brasil). Esses modelos foram enviados ao laboratório de prótese para o enceramento (Figura 2) e confecção de guia de silicone. A paciente informou o desejo de clarear seus dentes antes do tratamento estético, o qual foi realizado pela técnica de consultório.

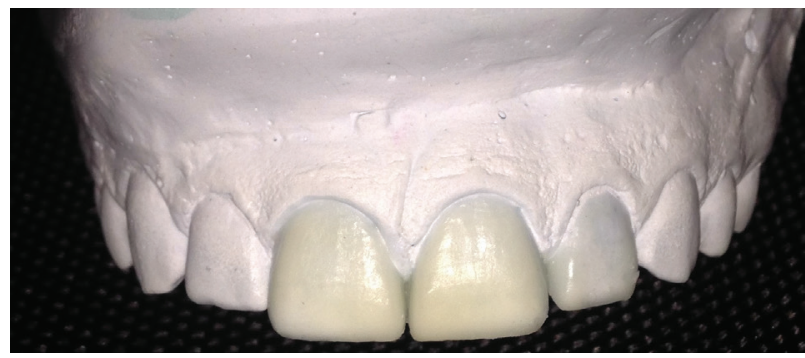

Figura 2. Enceramento do modelo com fechamento dos diastemas

O registro de cor inicial foi realizado com auxílio de escala de cor (Vita Classical, Wilcos, Brasil), sob luz natural, atribuindo cor A3. Foram feitas três sessões de clareamento dental, semanalmente, com peróxido de hidrogênio 35\% (Whiteness HP MAXX, Fgm, Brasil) de acordo com as instruções do fabricante (Figura 3). A cor final estabelecida foi A1.

Quatorze dias após a conclusão do clareamento, a paciente retornou à clínica para a confecção das restaurações. O guia de silicone de condensação laboratorial (Zetaplus, Zhermack, Brasil) foi testado e colocado em posição sob o arco da paciente, servindo como matriz para a confecção da porção palatal da restauração para tornar mais previsível a reconstrução dessa região dos elementos 11, 21 e 22. Realizou-se profilaxia com auxílio de pedra-pomes e escova de Robson. O isolamento absoluto modificado na região anterior foi confeccionado de pré-molar a prémolar. O condicionamento total no esmalte foi feito durante 30 segundos, com ácido fosfórico a $35 \%$ e lavado pelo mesmo tempo (Figura 4).

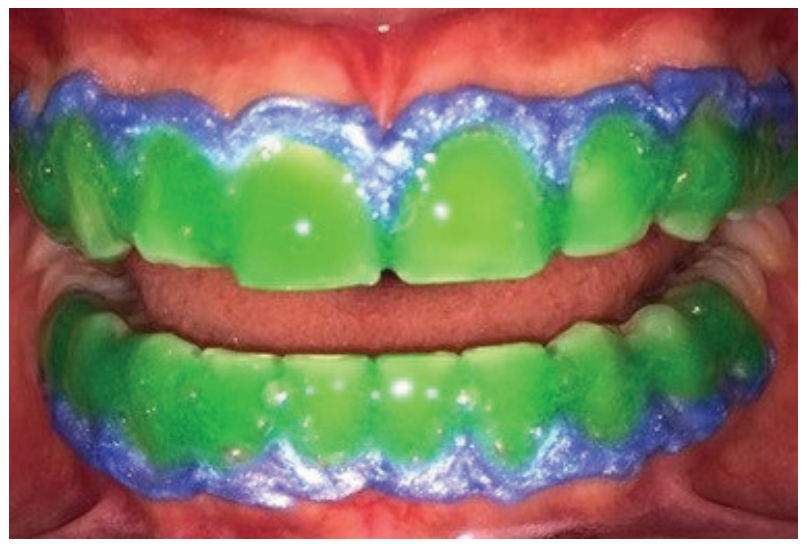

Figura 3. Clareamento no consultório com peróxido de hidrogênio a $35 \%$

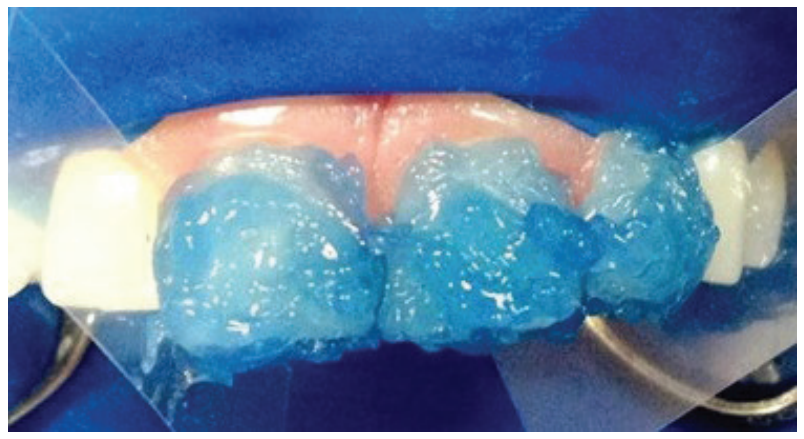

Figura 4. Condicionamento ácido total do esmalte nos elementos 11,21 e 22

O sistema adesivo (Single Bond, 3M Espe, Brasil) foi aplicado e fotopolimerizado por 20 segundos. Com o guia de silicone posicionado, os incrementos de resina composta foram inseridos com auxílio de espátula de inserção metálica. A resina utilizada foi $A 1$ de corpo e esmalte (Resina Filtek Z350 XT, 3M ESPE). Iniciou-se a restauração por meio da inserção da resina composta correspondente ao esmalte 
palatino, com o guia posicionada, onde o incremento foi fotopolimerizado (Figura 5).

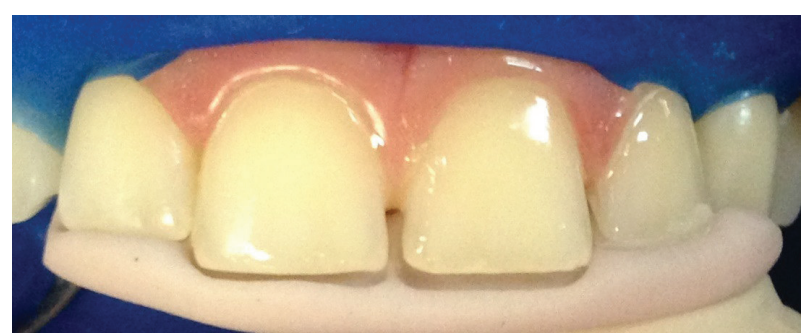

Figura 5. Inserção de resina composta na face palatina com o guia de silicone no incisivo lateral

Após a remoção do guia de silicone, foi verificado se a camada de resina presente estava sem falhas e iniciou-se a inserção de resina composta referente à dentina e esmalte vestibular, reproduzindo a forma dental conforme o enceramento diagnóstico. A remoção dos excessos grosseiros, com pontas diamantadas multilaminadas finas e ultrafinas em alta rotação, foi realizada. Verificaram-se os contatos oclusais em máxima intercuspidação habitual, protusiva, lateralidade esquerda e direita.

Posteriormente ao acabamento, o polimento da resina composta foi realizado, em sequência, com borrachas de silicone (Enhance, Dentsply, Brasil), discos de feltro associados à pasta diamantada, para a obtenção adequada de lisura superficial e textura das restaurações. A paciente relatou satisfação com o aspecto final do seu sorriso (Figura 6).

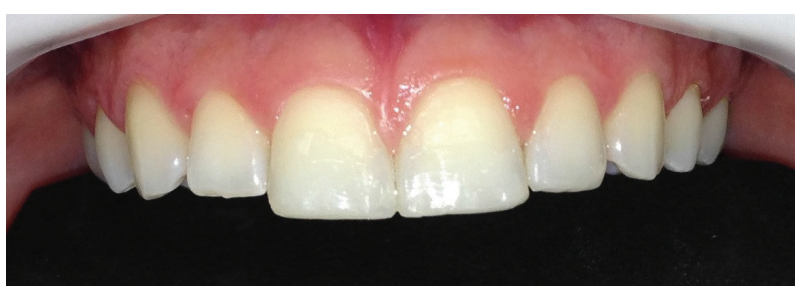

Figura 6. Aspecto final do sorriso após o fechamento de diastemas com resina composta

\section{Discussão}

A presença de diastemas na região de incisivos centrais e laterais superiores é considerado um fator antiestético prejudicial do ponto de vista social, pois podem comprometer a estética do sorriso substancialmente em função de sua extensão. ${ }^{7}$ Um sorriso atraente tem sido o foco de atenção da sociedade moderna. Cada vez mais, pacientes anseiam por uma aparência jovial e natural. A expectativa e vontade de ser mais desejado aos olhos do próximo é uma razão pela qual os pacientes procuram os consultórios odontológicos.

Como a procura por um sorriso perfeito e padrões estéticos ideais tem aumentado nos últimos anos, a ciência odontológica desenvolveu novos materiais, técnicas e procedimentos que atendemaos valores e exigências dos pacientes. ${ }^{8}$ Nesse sentido, a presença de diastemas no setor anterior pode marcar negativamente a aparência de um sorriso.

As etiologias desses espaços interdentais variam, podendo eles ser ocasionados por genética, discrepâncias no tamanho dental, ausência de dentes, trespasse horizontal excessivo, giroversões, angulações inadequadas entre dentes contíguos, presença de freio lingual com inserção baixa, presença de dentes supranumerários ou problemas periodontais. $^{7}$

Nos dias atuais, as indicações dos procedimentos adesivos variam, dependendo de cada caso. Não existe mais a possibilidade de indicações e contraindicações autoritárias e seguras. Hoje, quem define a indicação é o cirurgião-dentista, em vista de cada situação clínica e com base nos conhecimentos científicos.

A correção e o fechamento dos diastemas interincisivos por meio de coroas totais e/ ou facetas de porcelana são procedimentos restauradores considerados indiretos que, apesar de proporcionarem excelentes resultados estéticos e funcionais, são procedimentos invasivos, por necessitarem de um desgaste do tecido dental sadio, além disso, possuem a desvantagem de ter um custo financeiro alto para o paciente. , $^{6,9}$ 
Já a opção do tratamento ortodôntico é indicada somente em casos em que os espaços interdentais são mais extensos, o que ocorre devido à movimentação dental em todo o arco, distribuindo melhor o espaço. É um tratamento que requer mais tempo para o resultado final e mais dedicação do paciente. ${ }^{10}$ Entre as opções restauradoras, a realização de procedimentos diretos é considerada minimamente invasiva, além de desfrutar de grande popularidade. As restaurações e facetas em resina composta são famosas por sua excelente estética, longevidade aceitável e relativo baixo custo. A opção do fechamento de diastemas com resinas compostas apresenta grandes vantagens: técnica rápida, segura e eficaz, menor custo em relação às cerâmicas, dispensam etapas laboratoriais, não requerem provisório, sendo uma técnica reversível caso não se tenha alcançado o resultado estético desejado..$^{9,11}$

Entretanto, o sucesso clínico está diretamente relacionado ao material restaurador de escolha, a técnica a ser utilizada, bem como, a habilidade do profissional. Para que ocorra o sucesso funcional e estético das facetas em resina composta, o cirurgiãodentista deve saber diagnosticar e fazer o correto planejamento do caso clínico. Precisa também compreender e ter noções básicas dos princípios funcionais dos sistemas adesivos e as resinas compostas lançadas no mercado na atualidade. A dentística restauradora estética requer paciência, observação, perfeccionismo e a aplicação correta das técnicas e protocolos. $^{12,13}$

A matriz de silicone que é utilizada para realizar a redução ou o fechamento de diastemas e recontornos cosméticos pode ser obtida a partir de um ensaio restaurador diagnóstico, por meio de uma moldagem prévia da arcada do paciente. Essa matriz vai funcionar como um guia, que pode ser feito diretamente na boca ou a partir de um modelo de gesso. Esse é um método útil para se determinar a dimensão das restaurações adesivas a serem realizadas, assim como a exata posição da superfície lingual e incisal dos dentes anteriores. A partir do guia de silicone, o dente é reconstruído por meio da inserção de resina composta no espaço designado no enceramento, permitindo que a reconstrução dos dentes seja, além de mais eficiente, mais rápida. ${ }^{7,14}$

O acabamento e o polimento imediatos eram contraindicados no passado, devido à expansão higroscópica das resinas compostas, ${ }^{6,14}$ em contrapartida, o atraso do procedimento pode causar um aumento na microinfiltração e, atualmente, é sabido que não há qualquer efeito prejudicial à restauração quando essas etapas são realizadas na mesma sessão clínica em relação ao polimento tardio, ${ }^{13}$ portanto, no presente caso, optou-se por realizar o acabamento e o polimento imediatamente após a confecção da restauração.

O ajuste oclusal é indispensável após o término das restaurações, sem a necessidade de remover o dente de oclusão, porém removendo-se pontos de contato prematuros e possíveis interferências. ${ }^{13,15}$ Assim, foi realizada a análise oclusal estática e dinâmica da paciente, para permitir a longevidade e o sucesso do tratamento.

\section{Considerações finAIS}

Os tratamentos diretos para o fechamento de diastemas anteriores destacam-se por reduzido tempo de trabalho e baixo custo, apresentando resultado imediato, portanto, a realização de facetas diretas de resina composta mostrou-se eficaz no restabelecimento dos aspectos funcionais e estéticos do sorriso da paciente. 


\section{REFERÊNCIAS}

1. Andreiwolo R., Veiga W., Miragaya L., Dias $\mathrm{KRHC}$. Fechamento de diastema com coroas de alumina densamente sinterizadas. Rev Bras Odontol., 2011, jan./jun.; 68(1): 81-4. http:// revista.aborj.org.br/index.php/rbo/article/viewFile/256/222

2. Ubaldini ALM, Benetti AR, Furquim LZ, Pascotto RC. Abordagem interdisciplinar para o fechamento de diastemas associados à discrepância de Bolton. R Dental Press Estét. Maringá: 2009, out./nov./dez.; 6(4): 80-7. https://issuu. com/dentalpress/docs/v6n4

3. Goyatá FR, Coelho AJM, Oliveira RS, Ferreira TZF. Fechamento de diastema com resina composta microhíbrida. R Dental Press Estét. Maringá: 2007, out./nov./dez.; 4(4): 101-108. https://www.ufpe.br/ijd/index.php/exemplo/article/viewFile/108/116

4. Simões MP, Albino LGB, Reis AF, Rodrigues JA. Restaurações estéticas conservadoras em dentes anteriores. R Dental Press Estét., 2009; 6(1): 90-101.

4. Pucci CR, Barcellos DC, Paschotto DR, Borges $A B$, Torres CRG, Huhtala MFRL et al. Variação do guia de silicone em restaurações anteriores: relato de caso clínico. Int J Braz Dent., 2009 jan./mar.; 5(1): 34-41.

6. Conceição EN. Dentística - Saúde e Estética, 2. ed. Porto Alegre: Artmed; 2007.

5. Schwarz V., Simon LS, Silva SA, Ghiggi PC, Cericato $\mathrm{GO}$. Fechamento de diastema com resina composta: relato de caso. J Oral Invest., 2013; 2(1): 26-31.

6. Chimeli TBC, Pedreira PRV, Souza TCP, Paula LM, Garcia FCP. Tratamento restaurador de diastemas anteriores com restaurações diretas em resina composta: relato de caso. Rev Dent on line, $2011 ; 10(20)$ : $54-7$.

7. Araújo EP, Rocha Filho LA, Brum GT, Caldo-Teixeira AS. Fechamento de diastemas com restaurações diretas de resina composta - relato de caso clínico. Rev Gestão \& Saúde, 2009; 1(3): 33-8.

8. Wu CCL, Wong RWK, Hägg U. A review of hypodontia: the possible etiologies and orthodon- tic, surgical and restorative treatment options - conventional and futuristic. HKDJ 2007; 4(2): 113-21.

9. Mangani F., Cerutti A., Putignano A., Bollero R., Madini L. Clinical approach to anterior adhesive restorations using resin composite veneers. Eur J Esthet Dent., 2007; 2(2): 188-209. https:// www.researchgate.net/publication/26717768_ Clinical_approach_to_anterior_adhesive_restorations_using_resin_composite_veneers

10.Lamenha, EGR, Guimarães RP, Silva CHV. Diastema mediano superior: Aspectos etiológicos. Int J Dent., 2007 jan./mar.; (6)1: 2-6.

11. Silva AF, Lund RG. Dentística Restauradora: Do Planejamento à execução, 1. ed. Rio de Janeiro: Editora Santos; 2016.

12. Baratieri LN, Monteiro Júnior S., Melo TS, Ferreira KB, Hilgert LA, Schlichting LH et al. Odontologia Restauradora: Fundamentos e técnicas, v. 1. São Paulo: Editora Santos; 2013.

13. Machado AW, Ambrosio AR, Caldas SGFR, Gandini Junior LG. Orthodontic treatment of a midline diastema related to mesiodens and thumb-sucking habit. Rev Odonto Ciênc., 2010; 25(3): 314-8.

\section{Dados das Autoras}

\section{Micaela Lorena Raposo Seixas Guerra}

Graduada em Odontologia pela Universidade Nilton Lins. Manaus-AM - Brasil. mikaellaseixas@hotmail. com

\section{Gisely Naura Venâncio \\ Doutoranda em Clínicas Odontológicas pela Faculdade São Leopoldo Mandic. Campinas/SP - Brasil.ginaura@gmail.com}

\section{Carolina Rocha Augusto}

Doutoranda em Clínica Odontológica pela Universidade Federal do Rio Grande do Sul. Porto Alegre/RS - Brasil. carolinarochaaugusto@gmail. com

Submetido em: 08-07-2016

Aceito em: 14-02-2017 